\title{
PERFIL DEL PROFESOR Y DE LA CLASE UNIVERSITARIA EN CUBA: resultados de un estudio de caso
}

\author{
Maricela González Carmenate* \\ Orlando Fernández Aquino** \\ Roberto Valdés Puentes***
}

RESUME: En el presente artículo se traza el perfil del profesor y de la clase universitaria a partir de los documentos de política educativa del Ministerio de Educación Superior de Cuba/MES-Cuba y, a la par, se coteja con los resultados de un estudio de caso realizado en tres carreras del Centro Universitario de Sancti Spiritus, en el cruce de los años lectivos 2005-2006 y 2006-2007. El estudio focaliza el trabajo en la sala de aula, mediante la observación de 165 clases, lo cual se complementa con encuestas aplicadas a estudiantes y profesores. La discusión de los datos hace aflorar las incongruencias existentes entre la realidad de lo que acontece en el aula y el trazado de la política -incluido el perfil del profesorado-, y permite a los autores importantes consideraciones sobre la clase y la necesidad de operar un salto cualitativo en este privilegiado espacio institucional -tantas veces descuidado-, en el que se concretan las políticas, las prácticas pedagógicas y la formación de los egresados.

Palabras claves: perfil del profesor, clase universitaria, políticas educativas.

\section{PERFIL DO PROFESSOR E DA CLASSE UNIVERSITÁRIA EM CUBA: RESULTADOS DE UM ESTUDO DE CASO}

RESUMO: No presente artigo, traça-se o perfil do professor e da classe universitária com base nos documentos da política educativa do Ministério da Educação Superior de Cuba/MES-Cuba e, ainda, cotejam-se esses dados com os resultados de um estudo de caso realizado em três carreiras do Centro Universitário de Sancti Spiritus, nos anos letivos 2005-2006 e 2006-2007. O estudo focaliza o trabalho em sala de aula, por meio da observação de 165 classes, e se complementa com sondagens aplicadas a estudantes e professores. A discussão dos dados faz aflorarem as incongruências existentes entre a realidad do que acontece na aula e as propostas da política -incluindo o perfil do professorado-, e permite aos autores importantes consideraçõs sobre a classe e a necesidade de dar um salto qualitativo nesse privilegiado espaço institucional - tantas vezes desasistido-, no qual se concretizam as políticas, as práticas pedagógicas e a formação dos egressos. Palavras-chave: Perfil do Professor; Classe Universitária; Políticas Educativas.

* Master en Educación Superior por el Centro Universitario de Sancti Spiritus/CUSS, Cuba. E-mail: maricela@suss.co.cu

** Doctor en Ciencias Pedagógicas por el Instituto Superior Pegagógico Félix Varela, de Villa Clara - Cuba.E-mail: ofaquino@gmail.com

*** Doctor en Educación por la Universidade Metodista de Piracicaba (UNIMEP). E-mail: robertopuentes@faced.ufu.br 


\section{INTRODUCCIÓN}

En el 2004 se dio inicio al proyecto "Formación continua de profesores universitarios: desempeño docente, trabajo metodológico y categorización docente". ${ }^{1}$ Desde entonces los autores del presente artículo hemos sentido una preocupación creciente por los problemas que tienen que ver con la formación pedagógica del profesor universitario y las relaciones que ello guarda con lo que objetivamente ocurre en la sala de aula.

La práctica investigativa en el área de la formación docente, la observación atenta de la realidad, el ejercicio de la docencia en diferentes cursos y la revisión de la literatura nos han permitido vislumbrar una posible fisura existente entre el perfil del profesor -y el trabajo didáctico que se realiza para mejorarlo-, y la existencia de una práctica profesional que no cubre las expectativas planteadas. No obstante -como se sabe- una cuestión es la suposición hipotética y otra su verificación en el campo investigado.

La focalización de la sala de aula como espacio y tiempo particulares en que se materializa el proceso de enseñanza-aprendizaje y los continuos descuidos que ese escenario sufre en la mayoría de las IES, donde cualquier eventualidad es más importante que ese momento de verdadera trascendencia, es también una preocupación que está demandando una reconceptualización a la luz de los avances de la sociedad del conocimiento.

La importancia de la clase como tema y problema de la ciencia didáctica se refleja, principalmente, en que notables investigadores les han dedicado tiempo y estudio en diferentes contextos histórico sociales. La constante movilidad existente entre los paradigmas educativos y las diversas concepciones actuantes sobre el proceso de enseñanza-aprendizaje vuelven sumamente complejo el tema, pues la clase es un hecho educativo resultante -casi siempre-, de la formación pedagógica y del sentido que el docente imprime a sus prácticas profesionales.

A fin de avanzar en el conocimiento de esa compleja realidad y de brindar algunas valoraciones con base en la investigación de campo -si bien necesariamente limitadas-, durante el primer semestre del curso 2006-2007 nos dimos a la tarea de trazar el perfil del profesor universitario cubano y, a la vez, realizar un estudio de caso en tres carreras de la Facultad de Humanidades del Centro Universitario de Sancti Spiritus, 
Cuba. Ello permitió hacer un corte sincrónico de lo que ocurre, realmente, en el proceso formativo de las carreras estudiadas y cotejarlo con la política educativa de esa misma etapa.

Para el estudio de caso se seleccionaron los cursos de Estudios Socioculturales, Comunicación Social y Bibliotecología e Información Científico Técnica, de la Facultad de Humanidades del Centro Universitario de Sancti Spiritus. Dicha selección se hizo intencionalmente, ya que se considera que en el contexto del Centro Universitario de Sancti Spiritus, esas tres carreras responden a un Departamento Docente cuyo trabajo metodológico o didáctico es de buena calidad y adecuado a las exigencias del actual proceso de universalización de la educación superior en Cuba. ${ }^{2}$

La investigación empírica se sustentó en los métodos de análisis de documentos, en la observación de clases y en encuestas a profesores y estudiantes.

\section{DESARROLLO}

\subsection{Relaciones entre la concepción del proceso de enseñanza-aprendizaje y el trabajo en la sala de aula}

La concepción que el docente se forma acerca del proceso de enseñanza-aprendizaje influye de manera decisiva en sus ideas sobre la clase, en su manera de concebirla, dirigirla y evaluarla. Su noción sobre cómo debe transcurrir ese proceso es parte esencial de la formación del docente, de su motivación, de su responsabilidad profesional y de sus actitudes pedagógicas para dirigir el aprendizaje.

En una concepción tradicional del proceso pedagógico a éste se le concibe centrado en la figura del profesor, quien como regla, asume una actitud distanciada y autoritaria con respecto a los alumnos. Él se siente superior porque es el dueño de los conocimientos y no aprecia la urgencia de brindar atención a las necesidades de aprendizaje del alumno, ya sean individuales o grupales. En esta manera de concebir el proceso los docentes no implican a los alumnos en la búsqueda activa del conocimiento; más bien se anticipan a exponer sus razonamientos y enfoques sobre la materia que enseñan y a formular los juicios y conclusiones que debieran ser formulados por los estudiantes. 
En esta concepción del proceso docente los alumnos son considerados sujetos pasivos, que aprenden de manera reproductiva, y se les ofrecen escasas oportunidades para la reflexión, el intercambio y la discusión del contenido que se aprende. En este modelo "lo instructivo y cognitivo es separado de los afectivo y lo educativo, entendiéndose que las actividades educativas corresponden a las tareas extradocentes y extraescolares, no a la clase" (Castellanos, 2002, p. 16).

Como regla, los currículos carecen de pertinencia y significatividad personal y social de lo que se aprende. Los contenidos se ofrecen a partir de la lógica de las disciplinas independientes, generalmente descontextualizadas y obviándose las diferentes formas de las relaciones multidisciplinarias.

Otra es la concepción que el docente tiene del trabajo en la sala de aula si parte de un concepto del proceso que tiene su centralidad en el aprendizaje y el desarrollo integral de la personalidad de los estudiantes. $\mathrm{O}$ sea, si apoya su actividad profesional pedagógica en un proceso de enseñanza-aprendizaje desarrollador. Este tipo de proceso docente “...constituye la vía mediatizadora esencial para la apropiación de conocimientos, habilidades, normas de relación emocional, de comportamiento y valores, legados por la humanidad, que se expresan en el contenido de enseñanza, en estrecho vínculo con el resto de las actividades docentes y extradocentes que realizan los estudiantes" (Silvestre; Zilberstein, 2002, p.16).

Puede afirmarse que existen tres posiciones respecto a la didáctica: una de ellas es propia de la década de 1940 (Beltrán, 1985; Nassif, 1985), que la consideró como una de las ramas de la pedagogía, reservándole a esta ciencia un papel instrumental. Autores más recientes (Cárdenas, 1991; Zuluaga, 1992) han considerado que la didáctica sustituye a la pedagogía, restándole a esta última su carácter de ciencia. Una tercera posición ha sido desarrollada por investigadores cubanos (Varela, 1995; Silvestre \& Zilberstein, 2002; Castellanos, 2002; Zilberstein, 2006). Estos autores consideran a la didáctica como una de las ciencias de la educación, dentro de las cuales la pedagogía es la ciencia integradora de todas ellas. Esta tendencia considera que "el objeto de estudio de la didáctica lo constituye el proceso de enseñanza-aprendizaje, en su carácter integral desarrollador de la personalidad de los alumnos y alumnas" (ZILBERSTEIN, 2006, p.33). Los investigadores cubanos citados se orientan dentro de este enfoque desarrollador, el cual asumimos en el presente trabajo. 
La didáctica desarrolladora se apoya en la tradición pedagógica cubana, desde Félix Varela y José Martí hasta los pedagogos actuales, y sustenta sus criterios en el enfoque bistórico cultural que tiene como sus principales pilares a autores como L. Vigotski, L. Klimbert, P. Ya. Galperin, A. N. Leontiev, entre otros. Este enfoque considera que la didáctica debe conducir el desarrollo integral de la personalidad del estudiante, lo cual se logra a través del proceso activo de apropiación de la experiencia histórica acumulada por la humanidad. "El proceso de enseñanza-aprendizaje no puede realizarse teniendo en cuenta solo lo heredado por el alumno, debe considerar que es decisiva la intervención sociocultural, lo que existe en la sociedad, la actividad, la socialización, la comunicación" (ZILBERSTEIN, 2006, p.35).

$\mathrm{El}$ aprendizaje desarrollador es aquel que integra las dimensiones instructiva, educativa y desarrolladora en un solo proceso concebido como totalidad. O sea, que el proceso de enseñanza-aprendizaje está llamado a un importante rediseño, en el que se garantice la interacción dinámica de los sujetos con el objeto de aprendizaje y de los sujetos entre sí (profesor-alumno, alumno-alumno), y en el cual se combinan acciones dirigidas a la instrucción, al desarrollo y a la educación del alumno.

Figura 1: Dimensiones del aprendizaje desarrollador

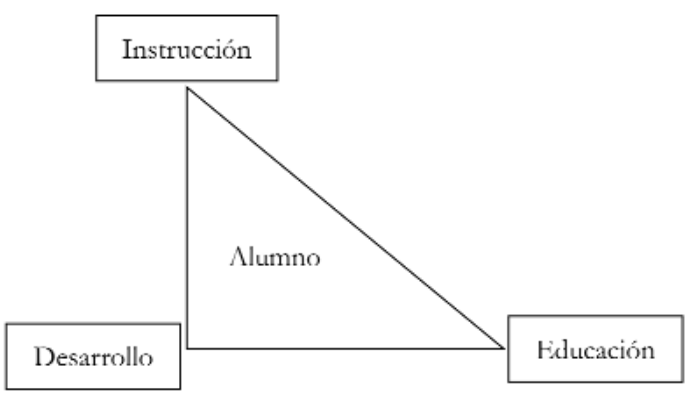

Al aprendizaje desarrollador se le ha conceptualizado de la siguiente forma: “... es aquel que garantiza en el individuo la apropiación activa y creadora de la cultura, propiciando el desarrollo de su auto-perfeccionamiento constante, de su autonomía y autodeterminación, en íntima conexión con los necesarios procesos de socialización, compromiso y responsabilidad social" (Castellanos, 2002, p. 33). 
Para que el aprendizaje sea desarrollador, deberá cumplir tres requisitos básicos:

a) Promover el desarrollo integral de la personalidad del educando, es decir, activar la apropiación de conocimientos, destrezas y capacidades intelectuales en estrecha armonía con la formación de sentimientos, motivaciones, cualidades, valores, convicciones e ideales. En otras palabras, un aprendizaje desarrollador tendría que garantizar la unidad y equilibrio de lo cognitivo y lo afectivo-valorativo en el desarrollo y crecimiento personal de los aprendices.

b) Potenciar el tránsito progresivo de la dependencia a la independencia y a la autorregulación, así como el desarrollo en el sujeto de la capacidad de conocer, controlar y transformar creadoramente su propia persona y su medio.

c) Desarrollar la capacidad para realizar aprendizajes a lo largo de la vida, a partir del dominio de las habilidades, estrategias y motivaciones para aprender a aprender, y de la necesidad de una autoeducación constante (Castellanos, 2002, p. 33).

La didáctica desarrolladora cubana -expuesta aquí en líneas generales- dialoga y tiene una sustentación psicológica y pedagógica muy cercana a las propuestas desenvueltas en Brasil por ANASTASIOU \& ALVES, 2003; LIBÂNEO, 2004; CUNHA, 2005; PIMIENTA \& ANASTASIO 2005; MASETTO, 2005, entre otros. La concepción de estos autores brasileños sobre el trabajo en la sala de aula y la consideración de la didáctica como un campo específico de investigación sorprende por su cercanía con el enfoque cubano aquí esbozado.

La visión intencionalmente dicotómica que hemos expuesto en el presente epígrafe, permite darnos cuenta mejor que según se tenga una concepción u otra del proceso de enseñanza-aprendizaje, se tendrá en consecuencia una noción determinada del trabajo en la sala aula. En otras palabras, la clase es una unidad cronotópica -para usar un término bajtiniano- más o menos terminada, del proceso de enseñanza-aprendizaje, pero que no puede confundirse con la totalidad de este. No obstante, el trabajo de dirección del aprendizaje que el docente concreta en la sala de aula es expresión de su visión del proceso, de su formación y habilidades profesionales. 


\subsection{Relaciones entre la concepción del proceso de enseñanza-aprendizaje y el perfil del profesor universitario}

Para comenzar cabe la pregunta siguiente: ¿Qué se ha de diseñar primero, el perfil del profesor, el modelo pedagógico o el proceso de enseñanza-aprendizaje?

Para nosotros resulta evidente que el tipo de profesor que se desea formar se subordina, en principio, al modelo pedagógico y, especialmente, a la concepción que se tenga del proceso de enseñanza-aprendizaje. Si se estuviera pensando en un modelo pedagógico autoritario, que reservara una función pasiva al alumno, donde el profesor sería prácticamente la única fuente de conocimiento y su función fuera la de mero trasmisor de información, entonces cabría formar a un profesor en los marcos de un modelo tecnológico, contenidista y de absoluta subestimación de la función docente. Si por el contrario, se piensa en un modelo pedagógico que privilegia el aprendizaje y la formación integral del alumno, habría que auxiliarse de una concepción del aprendizaje desarrollador y de un profesor adecuadamente preparado en el dominio del contenido didáctico de la enseñanza, capaz de situar al alumno en el centro de sus preocupaciones y artífice de su propia formación con la ayuda de sus pares, creando situaciones de aprendizaje que le permitan un desarrollo creciente. Aquí cambia el papel del profesor; ya no será más únicamente fuente de conocimiento, trasmisor de información, entidad lejana y totalitaria; será guía, mentor, partenaire, coadyuvante, acompañante, organizador, coevaluador. Y todo ello apoyado en la dialéctica existente entre lo cognitivo, lo afectivo y lo comunicativo.

En resumen, primero se debiera delinear el modelo pedagógico y el proceso de enseñanza-aprendizaje a través del cual aquel se va a concretar, y posteriormente el tipo de profesor que se necesita. Si lo anterior no se piensa como sistema y se subvierten los términos, se pudiera dar lugar a que cada una de las partes ande en dirección contraria, con el riesgo de no encontrarse jamás. Ello pudiera traer graves consecuencias para el sistema educativo.

\subsection{Perfil actual del profesor universitario cubano}

Entre los documentos de política educativa del MES-Cuba, probablemente el que mejor define la concepción del profesor universitario es el Reglamento para la aplicación de las categorías docentes de la educación superior 
(Resolución 128/2006). En el Capítulo II, Artículo 5, de dicha normativa se definen las funciones generales del personal docente de la siguiente manera:

a) educar para la formación de valores éticos y morales, convicciones personales, hábitos de conducta y personalidades integralmente desarrolladas que piensen y actúen creadoramente, aptas para construir la nueva sociedad y defender las conquistas de la Revolución;

b) realizar la planificación, ejecución y control del proceso docente de pregrado y postgrado en todas sus formas, de acuerdo con su categoría docente, c) desarrollar actividades metodológicas y de superación inherentes al proceso docente de pregrado y postgrado, hasta el nivel de actualización que requiera el desarrollo exitoso de las funciones correspondientes a su categoría;

d) elevar constantemente sus conocimientos pedagógicos, científico-técnicos y culturales;

e) realizar investigaciones, trabajos de desarrollo y de innovación tecnológica, así como servicios científico técnicos y de aplicación que contribuyan al desarrollo de las fuerzas productivas de la sociedad y al perfeccionamiento de la vida social en su conjunto; y

f) cumplir las regulaciones establecidas para el personal docente universitario (MES, 2006a, p.2).

En los artículos subsiguientes -6 al 9- se detallan las funciones específicas de las cuatro categorías principales: Profesor Titular, Profesor Auxiliar, Asistente e Instructor, las cuales conforman la pirámide de la titulación docente en el país. El examen de las funciones generales del personal docente y de las específicas que corresponden a cada una de las categorías ocupacionales permite concluir que este perfil se traza de acuerdo con:

a) La responsabilidad social y política del profesor universitario y su obligación de cumplir con las regulaciones establecidas para el personal docente de la educación superior.

b) La dualidad de funciones entre docencia e investigación que idealmente deben cumplir los profesores, sin que una de ellas tenga preeminencia sobre la otra.

c) La responsabilidad individual con la correcta planificación, ejecución y control del proceso docente de pregrado y de postgrado, en dependencia de la categoría que se ostente. Solo los Instructores no realizan docencia de postgrado, de acuerdo con la prescripción.

d) La participación y/o dirección de actividades metodológicas o trabajo didáctico en función del desarrollo profesional individual y colectivo. Los Profesores Auxiliares y Titulares tienen la mayor responsabilidad con los pla- 
nes de desarrollo didáctico de la institución.

e) La obligatoriedad de elevar constantemente sus conocimientos pedagógicos, científico-técnicos y culturales.

f) Las categorías docentes superiores de Profesor Auxiliar y Titular son requisitos para el desempeño de las responsabilidades académicas e investigativas más importantes.

g) Los Profesores Titulares, Auxiliares y Asistentes deben contribuir a la formación científico educativa, didáctica y científico-metodológica del personal con categorías docentes precedentes.

h) Se silencia el tema de la participación del profesor en la divulgación y promoción de la cultura científica y artística, aspecto importante relacionado con las funciones del profesor respecto a la extensión universitaria.

Sin duda alguna, el perfil que se traza aquí del profesor universitario cubano se sustenta en una valiosísima tradición y experiencia de la escuela cubana de educación superior. La estructura de categorías docentes se basa en el mérito académico y científico, y sus funciones responden a las realidades ocupacionales del sistema universitario.

Además del perfil trazado en el material que acabamos de glosar, otros documentos programáticos del MES-Cuba también delinean la figura del profesor universitario cubano. Así, por ejemplo, en un documento programático como "La universidad que queremos" (MES, 2004), se reconoce que "la calidad y competitividad de cualquier Institución de Educación Superior (depende de) sus recursos humanos y, en particular, su claustro" (p.16). Al mismo tiempo, se destaca que desde "la creación del Ministerio de Educación Superior en 1976 se ha trabajado en todas sus instituciones para garantizar los profesores altamente calificados e identificados con los principios de la Revolución, los que tienen como misión principal la formación integral de los especialistas de nivel superior que el país necesita" (p.16). Se insiste en que la envergadura de la transformación social emprendida en Cuba con el proceso de universalización lleva a los protagonistas a cambiar el modo de pensar, adecuándolo a las nuevas condiciones que traerán consigo dichos cambios.

En particular los profesores..., las autoridades académicas y los propios estudiantes, están obligados a despojarse de sus conceptos tradicionales y asumir los nuevos desde una posición abierta al cambio, con iniciativa y creatividad, porque solo de ese modo las transformaciones que se proponen podrán materializarse en la actividad práctica (MES, 2004, p. 8). 
Más adelante, al evaluar la idea rectora del enfoque integral de la formación, ${ }^{3}$ considerada la estrategia maestra principal del modelo universitario cubano, ahora aplicada al proceso de universalización, se explica que:

Un importante desafío del Enfoque Integral en la universalización lo constituye la preparación integral del claustro de profesores y especialmente de los adjuntos, para asumir el proceso de educación mediante la instrucción, en un tipo de curso en que la docencia es menos presencial. Particular importancia deviene en estas condiciones la existencia del tutor (...) ya que se trata de la figura que acompaña al estudiante durante toda su carrera, lo asesora y lo guía, e impide que se sienta abandonado, solo, desalentado (p.12).

En las nuevas condiciones de universalización de la educación superior se produce un rescate de la figura del tutor, por demás milenaria en la historia de la educación. ${ }^{4}$

El ejemplo personal, el liderazgo y la perseverancia del tutor con los estudiantes forman parte de las relaciones interpersonales que fluyen y se convierten en modos de actuación de los jóvenes universitarios, contribuyendo a la formación de una personalidad acorde con su futura vida profesional. De esta forma, los tutores deben convertirse en líderes académicos con una alta profesionalidad (...) Este proceso permite rescatar la condición de discípulo de su tutor, reconociendo a este último, no solo por sus cualidades profesionales, sino también por su liderazgo como educador (p.12).

Otros documentos de la política educativa del MES-Cuba, como son los objetivos estratégicos para cada año lectivo y los programáticos de las IES, permiten acopiar elementos que ayudan a ampliar la visión sobre el tipo de profesor que requiere el sistema, sobre todo porque establecen aspiraciones en el orden didáctico, organizativo, de infraestructura, etc., que tienen que ver con las funciones del profesor y con su papel en el proceso formativo. ${ }^{5}$

\subsection{Resultados del estudio de caso}

\subsubsection{Resultados de las visitas a clases}

Como se ha explicado en la Introducción, para realizar el estudio de caso se seleccionaron tres carreras del Centro Universitario de Sancti Spiritus. Ellas son: Estudios Socioculturales (ESC), Comunicación Social (CS) y Bibliotecología y Ciencias de la Información (BCI). De esos 
tres cursos solo ESC posee Curso Regular Diurno (CRD), las otras dos se dirigen desde la Sede Central de la Universidad pero solamente se cursan en las Sedes Universitarias Municipales (SUMs).

La tabla que sigue muestra un resumen de las clases observadas y evaluadas en dichas carreras, tanto en la Sede Central como en las SUMs. En la evaluación de cada clase participó un equipo pequeño de especialistas, integrado indistintamente por el vicedecano docente, la jefa de departamento, coordinadores de carrera (provinciales y municipales) y profesores principales de disciplinas. ${ }^{6} \mathrm{El}$ trabajo se organizó en equipos para buscar la mayor justeza posible en el análisis y la evaluación.?

\begin{tabular}{|c|c|c|c|c|c|c|}
\hline \multirow[t]{2}{*}{ Calificaciones } & \multicolumn{4}{|c|}{ SEDES } & \multirow[t]{2}{*}{ Total } & \multirow[t]{2}{*}{$\%$} \\
\hline & Central & $\%$ & SUMs & $\%$ & & \\
\hline Con 5 ptos. & 2 & 9,5 & 47 & 32,6 & 49 & 29,6 \\
\hline Con 4 ptos. & 14 & 66,6 & 77 & 53,4 & 91 & 55,1 \\
\hline Con 3 ptos. & 4 & 19 & 15 & 10,4 & 19 & 11,5 \\
\hline Con 2 ptos. & - & - & - & - & - & - \\
\hline No evaluadas & 1 & 4,7 & 5 & 3,4 & 6 & 3,6 \\
\hline Totales & 21 & 100 & 144 & 100 & 165 & 100 \\
\hline
\end{tabular}

Como se aprecia, se observó un total de 165 clases, de ellas 21 en el CRD y 144 en las SUMs. La tendencia muestra que el 55,1\% de ellas está evaluado de 4; el 29,6\% está evaluado de 5; el 11,5\% de 3 y el 3,6\% son no evaluadas. No se registran clases de 2 ptos.

Según se muestra la propensión es positiva, pero la sospecha lleva a pensar que las no evaluadas son en la práctica de 2 ptos. Es posible calcular también que las calificaciones con nota 5 debieran reservarse únicamente para aquellas clases de excelencia. No hay que dudar que las diferencias de preparación y de criterios metodológicos entre los evaluadores, puede introducir un sesgo en los resultados. De todas formas, lo verdaderamente importante es constatar las tendencias dominantes.

Para realizar un análisis de los indicadores de la Guía de Observación de las Actividades Docentes, se tomó como muestra aleatoria 34 aulas seleccionadas al azar. Dicha muestra estuvo conformada por 10 observaciones en el CRD; 15 en la Sede de Cabaiguán y 9 de la Sede de Jatibonico. La muestra representa el 20\% de las 165 evaluadas.

A partir de esa muestra fue posible detectar los indicadores más afectados en la dirección del proceso docente y realizar una valoración de 
los aspectos positivos y negativos observados en las clases. A continuación presentamos dicha síntesis.

Aspectos positivos de las classes observadas:

- De modo general, buena autopreparación de los docentes.

- Los profesores se expresan correctamente y usan el metalenguaje de su asignatura.

- Se utilizan, como tendencia, métodos que propician el aprendizaje activo y consciente.

- Se orientan adecuadamente los objetivos.

- Se utilizan esquemas lógicos, cuadros, resúmenes, láminas y vídeos que activan el proceso de enseñanza-aprendizaje.

- Se vela por el uso correcto del idioma materno.

- Se hacen adecuados comentarios bibliográficos y se orienta la bibliografía en las clases controladas.

- La mayoría de las clases poseen una organización y estructura metodológica efectivas.

- Se observa una mejora en el aprovechamiento de las potencialidades político- ideológicas y de formación de valores a través de la instrucción.

Aspectos negativos de las classes observadas

- Las salidas de las estrategias curriculares o ejes transversales (lengua extranjera, comunicación, NTIC, medio ambiente y otras) a través de la clase, son sumamente discretas, afectándose la interdisciplinariedad y la transdisciplinariedad.

- Como tendencia, persisten deficiencias con la correcta planificación y orientación del estudio independiente. Ello trasciende al control que después se realiza de las tareas de aprendizaje y a la deficiente aplicación del sistema de evaluación. Este aspecto influye también en actitudes inadecuadas de los alumnos y en sus inasistencias a clases.

- Como regularidad, existe un bajo aprovechamiento de las posibilidades que brindan las NTIC y del Programa Audiovisual. En ello están influyendo las carencias de infraestructura que presenta la universidad, tanto en las SUMs como en la Sede Central.

- La mayoría de los profesores no trabaja de forma ordenada y cuidadosa en el pizarrón, con el fin de servir de modelo de actuación a los estudiantes. 
- No se precisa, en general, la significación social y profesional de los contenidos que se trabajan en clases, lo que puede estar influyendo de manera negativa en la motivación y orientación profesional.

- En la organización y dirección de la clase se aprecia escasa personalización al asignar las tareas de aprendizaje, así como en la atención a las diferencias individuales en el proceso de su realización. En otras palabras, los profesores atienden más a los pequeños grupos o a la totalidad de este, que a los estudiantes de manera personalizada.

- No siempre las conclusiones parciales y finales de la clase reciben el tratamiento adecuado.

\subsubsection{Resultados de las encuestas aplicadas a los profesores}

En el momento de realización de la investigación, la carrera de ESC tenía un claustro de 171 docentes, la de CS, 123 y la de BCI, 20. Ese universo estaba compuesto por un total de 314 profesores, cifra que incluye los coordinadores. Se seleccionó una muestra aleatoria de 70 docentes, lo que representa el 22\% del universo, para la aplicación de la encuesta. La síntesis cualitativa de los criterios de los mismos es la siguiente:

Variable 1: Insuficiencias del trabajo de las asignaturas

- Algunos profesores todavía no contribuyen de modo efectivo a concretar el Proyecto Educativo y a lograr la integralidad en la formación de los alumnos.

- Existen deficiencias en el trabajo con enfoque interdisciplinario para la formación de habilidades generales.

- El diagnóstico de los estudiantes aún no posee toda la precisión y objetividad que se requiere para lograr su atención personalizada.

- Los materiales digitales que se entregan a las SUMs por parte de las Coordinaciones Provinciales de las Carreras, casi no se consultan por los estudiantes. Se convierten tan solo en textos para la autopreparación del docente.

- La no obligación de la asistencia a clases en modelos pedagógicos como el de la universalización atenta contra el autoaprendizaje y la calidad de la docencia.

- Existen dificultades con la "responsabilidad" en algunos estudiantes de la universalización. Ellos no tienen una buena actitud ante el estudio independiente y esto obstaculiza la aplicación de métodos activos. 
- Existe falta de sistematicidad en el estudio individual, así como necesidad de continuar desarrollando habilidades para la realización del estudio independiente, tanto en las SUMs como en la Sede Central.

- Persisten dificultades en el desarrollo de habilidades en el uso del idioma materno en cuanto a comunicación, redacción y la ortografía.

- La evaluación del aprendizaje, como tendencia, no se realiza de forma óptima, ya que hay baja exigencia en las comprobaciones sistemáticas y las evaluaciones parciales y finales carecen de la integralidad necesaria, lo que puede estar afectando el cumplimiento de los objetivos de los programas.

- Los métodos, medios y estrategias de dirección de los aprendizajes empleados por los docentes, en general, todavía no se encuentran a la altura requerida para lograr el protagonismo de los estudiantes y su autoformación.

•Existen insuficiencias en la planificación y ejecución de acciones concretas para el logro de los objetivos educativos, desde la clase.

\section{Variable 2: Infraestructura}

- Se utilizan de forma limitada las potencialidades de las NTIC en la autopreparación y el desarrollo del estudio independiente de los alumnos, pues no existe una infraestructura en los laboratorios del CUSS y de las SUMs que lo permita.

- Las averías que continuamente se producen en la red afectan la calidad del proceso docente, sobre todo en la elaboración de materiales de apoyo para los estudiantes y la localización de información actualizada por parte de estos en diferentes páginas $W e b$. Esta dificultad se hace extensiva a los problemas de conectividad que sufren las SUMs.

- El Programa Audiovisual no se implementa ya que no existe una infraestructura que facilite la proyección de videos, filmes, visualización de obras plásticas, etc. Esta carencia afecta, en especial, a asignaturas como Música Cubana, Arte Cubano, Cine Cubano y Apreciación del Arte.

- Solo un número limitado de asignaturas se encuentran montadas en páginas $W e b$ o en plataformas interactivas.

- Aunque todas las asignaturas tienen cobertura bibliográfica, todavía el módulo es insuficiente. Estas carencias han sido suplidas, en parte, por materiales docentes elaborados por los profesores.

- El módulo bibliográfico de las asignaturas es insuficiente, aun- 
que se ha elaborado por parte de los profesores una serie de folletos actualizados y dossier que han atenuado las limitaciones de la bibliografía básica y sus niveles de actualización.

- En el semestre estudiado no se contó con los materiales del At Your Pace, lo que ha impedido el cumplimiento de la Estrategia de Idioma Extranjero. La misma se encuentra afectada, además, por la escasez de literatura en lengua extranjera en las diferentes carreras, amén de las insuficiencias de formación idiomática que presenta un número considerable de profesores.

- Las condiciones de local de los docentes no son las deseadas, pues no existe privacidad y el mobiliario es insuficiente.

- En la Sede Central ha habido una mejora sustancial de las condiciones de las aulas, pues han sido beneficiadas con pintura e iluminación.

- En las salas de aulas no se cuenta con televisores, ni videos, ni grabadoras. Tampoco con retroproyectores o proyectores de vistas fijas. Se carece de un stock suficiente de cassetes vírgenes para grabar películas y videos educativos.

\subsubsection{Resultados de la encuesta a estudiantes}

La carrera de ESC tiene una matrícula total de 1101 estudiantes, en CS es de 951 y en BCI de 200. O sea, que el universo es de 2252 estudiantes. De ese total se escogió una muestra aleatoria simple de 375 alumnos para la aplicación de encuestas y entrevistas grupales. A continuación transcribimos una síntesis de esos resultados.

- El 80\% de la muestra considera que recibe una docencia de calidad.

- El 60\% exigen más rigor y dedicación a la práctica laboral y mayores relaciones con las instituciones laborales del área de formación de la carrera.

- El 80\% considera insuficiente la preparación de algunos profesores.

- El 31\% demanda más actividades prácticas y menos teoría.

- El 30\% considera obsoleta una parte de la literatura existente.

- El 95\% valora positivamente a los profesores que confeccionan y disponen materiales de apoyo y dossier.

- El 40\% exige mayor participación en tareas investigativas y de 
mayor impacto social.

- El 80\% se muestra ávido por participar en actividades culturales y recreativas de calidad.

- El 80\% valora como muy positivo compartir el aula y la experiencia cultural con estudiantes de otras nacionalidades.

- El 80\% considera deficiente el servicio prestado por la red universitaria. Valoran altamente necesario el acceso a las NTIC para su formación profesional y cultural general.

\subsection{Discusión de los resultados}

Para la discusión de los resultados del presente estudio nos apoyamos alternativamente en el Informe Anual de Balance que realiza la Dirección de Formación de Profesionales del MES-Cuba, en algunos de sus documentos de política educacional, tales como prioridades, objetivos estratégicos, etc., así como en las prioridades, objetivos y documentos que rigen la política de formación de profesionales en el Centro Universitario de Sancti Spiritus (CUSS) Cuba. (Ver bibliografía). Ello permite cotejar los datos de investigación con la política a nivel nacional y a nivel de IES. La discusión se realiza sobre la base de cuatro prioridades de la de formación de profesionales: 1) enfoque integral de la formación; 2) actividad investigativo laboral de los estudiantes; 3) infraestructura; 4) trabajo metodológico o didáctico.

\subsection{Enfoque integral de la formación desde la instrucción}

Entre las prioridades del CUSS, para el curso 2006-2007, se propone que la satisfacción de los estudiantes con el proceso de formación sea, al menos, de un $90 \%$ (CUSS, 2006, p.1). En el Informe de Balance de la Dirección de Formación de Profesionales se afirma que se han obtenido "avances en la labor educativa desde el contenido de las disciplinas en todos los CES” y que a nivel nacional el $97 \%$ de los estudiantes presentan satisfacción con las cualidades de los profesores como educadores (MES, 2006b, p. 1). En las carreras estudiadas, el 80\% de la muestra considera insuficiente la preparación de algunos profesores y solo un número reducido valora ser de calidad la docencia que recibe. $O$ sea, que en este caso la realidad estudiada no se corresponde con los parámetros nacionales, ni con las aspiraciones del CUSS.

Entre las prioridades de la Dirección de Formación de Profesionales del MES-Cuba, para el curso 2005-2006, se propone: 
"Fortalecer la atención personalizada a los estudiantes. Asegurar un diagnóstico de mayor calidad, que identifique con precisión las diferencias individuales, tanto en el plano educativo como en el instructivo" (MES, 2005a, p.1). El Informe de Balance del MES-Cuba, sobre el mismo período, considera que, a nivel nacional, se ha logrado un "diagnóstico más integral de las necesidades educativas e instructivas de los estudiantes, así como una instrumentación y control más estable que en cursos anteriores” y que hay un "93 \% de satisfacción de los estudiantes con su participación en la elaboración y desarrollo de su proyecto educativo” (MES, 2006b, p. 1); mientras que en el caso estudiado se detectan profesores que no contribuyen de modo efectivo a concretar el Proyecto Educativo y a lograr la integralidad en la formación. El diagnóstico de los estudiantes aún no posee toda la precisión y objetividad que se requiere para lograr su atención personalizada. En resumen, se aprecia disparidad entre las aspiraciones y resultados a nivel nacional y la realidad de las carreras estudiadas.

En el caso examinado se detecta la persistencia de dificultades en el desarrollo de habilidades en el uso del idioma materno en cuanto a comunicación, redacción y ortografía, lo que coincide con el balance nacional, donde se plantea que "todavía se presentan insatisfacciones de los estudiantes en algunos centros, derivadas de una inadecuada comunicación, que limitan también la efectividad de la labor educativa" (MES, 2006b, p. 1).

El balance del MES reconoce que "aun no se logra que la figura del tutor abarque todo el espectro de años y carreras de los cursos diurnos" y manifiesta insuficiencias en la efectividad de los tutores, en su preparación y atención por parte de las IES para que estos puedan desarrollar su función como presupone el modelo pedagógico de la universalización (MES, 2006b, p. 1). Sin embargo, en el estudio realizado el tema es silenciado por profesores y estudiantes, lo que puede interpretarse como falta de preocupación por el mismo.

\subsection{Actividad investigativo laboral de los estudiantes}

El MES-Cuba se plantea: "Continuar avanzando en la elevación de la calidad de la práctica laboral, a partir del mejoramiento de la organización de la actividad, la atención de profesores y profesionales, las guías de trabajo, los contenidos de trabajos de curso y de diploma, entre otros (...)" (MES, 2005a, pp. 2-3). En el informe de Balance del 2005-2006 se afirma que el $90 \%$ de los estudiantes siente satisfacción con su actividad inves- 
tigativo-laboral y reconoce que "en algunos CES la satisfacción de los estudiantes con la práctica laboral sigue siendo baja y susceptible de mejorar" (MES, 2006b, p. 1). Esto último se corresponde con el caso estudiado, ya que el 60\% de los estudiantes encuestados exigen más rigor y dedicación a la práctica laboral y mayores relaciones con las instituciones laborales del área de formación de la carrera. El sentimiento general es que su formación debiera ser más práctica que teórica, acorde con los modos de actuación de la profesión.

\subsection{Infraestructura. Su incidencia en el proceso formativo}

Con respecto al aseguramiento bibliográfico, el estudio revela que todas las asignaturas tienen cobertura bibliográfica, con lo cual se da cumplimiento a lo dispuesto en la política. No obstante, el 30\% de los alumnos encuestados considera obsoleta una parte de la literatura existente. Este es también un criterio dominante entre los profesores, pues, a pesar de que cada asignatura posee al menos un texto básico, no siempre es el mejor de los existentes y, en ocasiones, ya está desfasado con respecto al desarrollo de la ciencia.

En relación con la transformación de las asignaturas para asegurar un adecuado empleo de las NTIC, las prioridades para el 2005-2006 previeron transformaciones didácticas del contenido, el óptimo empleo de los software profesionales, laboratorios virtuales y otras herramientas similares disponibles para la asignatura, el diseño adecuado de sitios Web, plataformas, o similares, acciones que aseguraran adecuados niveles de interactividad, así como el control sistemático del empleo de los estudiantes de todos esos recursos (MES, 2005a, pp. 2-3). No obstante, el estudio reveló que solo un número limitado de asignaturas se encuentran montadas en páginas Web o plataformas interactivas y que el uso de laboratorios virtuales es inexistente. La insatisfacción de profesores y estudiantes con los servicios que presta la red universitaria y las continuas averías que presenta es considerablemente alta ( $80 \%$ de insatisfacción en los estudiantes). Las clases observadas y las encuestas a profesores confirman el uso limitado de las NTIC para el desarrollo del proceso de formación. Esto contradice lo propuesto entre las prioridades del área de Formación de Profesionales del CUSS, al aspirar a lograr niveles superiores de virtualización de las asignaturas y a elevar la satisfacción de los estudiantes con el aseguramiento bibliográfico sobre el 85 \% (CUSS, 2006, p.1). 
Entre las prioridades del MES-Cuba se planteó: "Asegurar que en todas las carreras tenga lugar un perfeccionamiento de la estrategia de idioma inglés, a partir de la introducción del sistema At Your Pace" (MES, 2005a, p. 2). En el semestre estudiado no se contó con los materiales del At Your Pace, lo que ha impedido el cumplimiento de la Estrategia de Idioma Extranjero. La misma se encuentra afectada, además, por la escasez de literatura en lengua extranjera en las diferentes carreras, amén de las insuficiencias de formación idiomática que presenta un número considerable de profesores. Lo anterior es reconocido por el MES cuando plantea que: "En varios CES la estrategia de idioma inglés sigue reflejando deficiencias que se revelan en la baja satisfacción de los estudiantes con su proceso de formación" y que "en la aplicación de la serie At Your Pace, se presentaron algunas deficiencias en su aseguramiento material y en la preparación de los estudiantes para los encuentros, que deben ser atendidas y resueltas" (MES, 2006b, p. 3).

La carencia de otros medios como proyectores de vista fija, videos, retroproyectores, etc., también afecta la calidad de la formación de los profesionales en las carreras estudiadas.

Se reconocen mejoras en las condiciones de trabajo de la Sede Central y las Municipales, no obstante persisten las dificultades con el acondicionamiento y hacinamiento en los locales de los profesores, la falta de privacidad y de recursos informáticos para los docentes.

\subsection{Trabajo metodológico o didáctico}

Entre las Prioridades de la Dirección de Formación de Profesionales para el Curso 2005-2006 se propone lo siguiente: “Atender sistemáticamente el trabajo metodológico... en función de la gestión del proceso de formación, con énfasis en la preparación de los profesores a tiempo parcial y de los tutores (...) y en particular en como conducir adecuadamente el autoaprendizaje y la autoeducación (...)" , así como “el diseño de los sistemas de evaluación con un enfoque más cualitativo e integrador, basados en el desempeño del estudiante durante el proceso de formación" (MES, 2005a, p. 2, 3).

Por su parte, entre los criterios de medida del CUSS para el 2006-2007 se plantea que: "El trabajo metodológico integra a todos los profesores a tiempo completo y a tiempo parcial (...) Se prioriza el empleo de métodos semipresenciales y se logran niveles superiores en la atención 
a las dificultades que presentan los estudiantes en el aprendizaje" (CUSS, 2006, p. 1).

Mientras, en las clases observadas durante el estudio se evidencian afectaciones del trabajo con las estrategias curriculares, con el desempeño de los profesores para la planificación, orientación y evaluación del estudio, con el uso correcto de métodos, medios y estrategias de dirección del aprendizaje, con el destaque de la significación social y profesional de los contenidos, con la personalización y evaluación del aprendizaje, etc.

Por su parte, el Balance del Curso 2005-2006, a nivel nacional, plantea que "el $97 \%$ de los estudiantes está satisfecho con el nivel de exigencia y rigor del proceso de formación en su carrera" y que "el $94 \%$ de los estudiantes está satisfecho con la preparación profesional recibida" (MES, 2006b, p. 3). Sin embargo, la constatación realizada durante la experiencia muestra que solo el $80 \%$ de los estudiantes considera que recibe una docencia de calidad; el $60 \%$ exige más rigor y dedicación a la práctica laboral, el 31\% demanda más actividades prácticas y menos teóricas, y el $80 \%$ considera que debe tener más participación en actividades culturales de calidad como complemento a la formación.

El informe de Balance del 2005-2006 reconoce atinadamente que "aunque hay avances en el curso (entiéndase año lectivo), el trabajo metodológico que se desarrolla en las sedes centrales aun no da respuesta... a las exigencias del modelo de formación de las SUM" y que "la labor de atención y asesoría de la DFP (Dirección de Formación de Profesionales) a los CES no logra aun la sistematicidad que demandan las profundas transformaciones que hoy tienen lugar en la educación superior" (MES, 2006b, p. 3).

En nuestra apreciación, la contradicción fundamental existente entre los resultados que arroja el estudio de caso, el perfil del profesor universitario y la política educativa trazada por el MES-Cuba radica, esencialmente, en que en el profesorado continúa primando una concepción tradicionalista del proceso de enseñanza-aprendizaje y a que, en consecuencia, las prácticas pedagógicas se perpetúan en esa dirección. Todo ello, a contrapelo del pensamiento pedagógico más avanzado -actualización del modelo de formación, mejora de la teoría sobre el proceso de enseñanzaaprendizaje, etc-, de un adecuado trazado de la política -incluido el perfil del profesor- y del loable esfuerzo que se realiza en muchas carreras universitarias para acompañar el proceso pedagógico mediante el trabajo 
metodológico o didáctico. El foco principal a partir del cual se irradian las dificultades es -para estos investigadores-, lo que acontece en la sala de aula, sin negar otros importantes espacios en que se concreta la formación de los profesionales.

\section{CONCLUSIONES}

Como se ha podido apreciar, el perfil del profesor universitario cubano se traza a partir de sus funciones generales y de las específicas asociadas a la categoría docente. Además de eso, las prioridades para cada curso escolar y otros documentos programáticos permiten ampliar y flexibilizar las funciones docentes, en relación con los contextos en que se desarrolla cada período lectivo. Hay que convenir en que las exigencias delineadas para el profesor universitario se avienen con el modelo pedagógico de la formación de profesionales en Cuba y con la concepción que el sistema tiene sobre las esencias de un proceso de enseñanza-aprendizaje desarrollador.

Otro aspecto de no menor importancia es la preocupación manifiesta del MES-Cuba por el desarrollo profesional continuo de su cuerpo docente, especialmente en los aspectos didácticos o metodológicos, para poder dar respuesta con calidad a las transformaciones que el sistema va exigiendo en cada nueva etapa de desarrollo.

La contrastación de los diversos aspectos que componen las dimensiones: enfoque integral de la formación desde la instrucción, actividad práctico laboral de los estudiantes, infraestructura y trabajo metodológico, contenidos en la política educativa del MES-Cuba en el cruce de los cursos 2005-2006 y 2006-2007, con los resultados encontrados en el estudio de caso realizado en tres carreras universitarias, evidencia numerosas insuficiencias en la concreción de la política. La mayoría de las incongruencias encontradas se deben principalmente a deficiencias en la formación de los profesores y a su desempeño en la sala de aula; y, en menor medida, a problemas de infraestructura y de recursos materiales, si bien estos últimos tienen un peso importante en la calidad del proceso formativo.

A juicio de los investigadores, los resultados del presente estudio no revelan necesariamente errores en el trazado de la política educativa. 
Debe convenirse en que los expertos del MES-Cuba tienen una visión objetiva de la realidad educacional del país -hasta donde es posible tenerla-, que son conscientes de los riegos aparejados al sistema de cambios que hoy lideran y que la política es siempre una aspiración, nunca un hecho. Los resultados de la constatación más bien alertan sobre la necesidad de desarrollo profesional de los profesores, sobre todo en las condiciones de cambio raigal en que se encuentra el sistema universitario cubano. Ese desarrollo profesional puede, sin dudas, acometerse desde varias direcciones, pero la fundamental ha de ser la de su acompañamiento por la vía del trabajo metodológico o didáctico. Sin acompañamiento de lo que sucede en la sala de aula, y del proceso formativo en general, difícilmente pueda concretarse el perfil del profesor y el de la política. Un elemento esencial para lograr la superación de preconceptos y hacer avanzar el desarrollo profesional ha de ser el análisis sistemático de las prácticas pedagógicas en los colectivos docentes, hasta convertir el procedimiento en método y estilo fundamental de la labor didáctica.

Quizás la principal evidencia del presente estudio sea la siguiente: sólo la voluntad estratégica de focalización de la clase universitaria como ambiente cronotópico y suigeneris en que se concretan las políticas educativas, las prácticas pedagógicas y la formación de los egresados; y, la comprensión de la necesidad, por parte del profesorado y de los gestores, de lograr un salto cualitativo profundo en su concepción y ejecución nos pondrá en el camino de alcanzarlo. La reconceptualización y flexibilización la clase universitaria -a tono con el contexto informacional, científico y cultural de nuestros días-; la actualización de las funciones de lo que hoy significa enseñar; y la búsqueda activa de estrategias capaces de colocar al alumno cada vez más ante nuevas situaciones de aprendizaje, son algunos de los caminos posibles para emprender esa interminable pero fructífera trayectoria. 


\section{REFERENCIAS}

ANASTASIOU, L. da G. C.; ALVES, L. P. (Org.) Processos de ensinagem na universidade: pressupostos para a estratégia de trabalho na aula. Joinville, SC: UNIVILLE, 2003.

AQUINO, O. F. A Formação docente, uma paixão inevitável. Revista Ícone Educaşão. Programa de Pós-graduação em Educação Centro Universitário do Triângulo, v. 12, no. 1, jan./jun. 2006, p. 07-13.

AQUINO, O. F.; PUENTES, R.V. Calidad del proceso de enseñanza-aprendizajeinvestigación en la educación superior: un modelo para su evaluación. In: Ícone Educação. Uberlândia: v.10, n.1 e 2, 2004, p. 207-234.

CASTELLANOS, D. et al. Aprendery enseñar en la escuela. La Habana: Pueblo y Educación, 2002.

CENTRO UNIVERSITARIO DE SANCTI SPIRITUS/CUSS. Objetivos 06-07 ARC Formación de Profesionales. Vicerrectorado Docente Educativo, 2006.

CUNHA, Maria I. (Org.) Formatos avaliativos e concepşão de docência. Campinas, SP: Autores Asociados, 2005.

HORRUITINER, P. La Universidad Cubana. El Modelo de Formación. La Habana: Félix Varela, 2006. (Versión digital)

KLINGBERG, L. et al. Didáctica General. Separata 2. La Habana: Pueblo y Educación, 1970.

LABARRERE, G.; VAlDIVIA, G. E. Pedagogía. Segunda reimpresión. La Habana: Pueblo y Educación, 2001.

LIBÂNEO, J. C. As políticas de formação de professores no contexto da Reforma Universitaria. Conferência. VII EPECO. Encontro de Pesquisa em Educação do Centro-Oeste, Goiânia - GO, Anais do VII EPECP, 9 a 11 de junho de 2004.

MASETTO, M. T. (Org.) Docência na universidade. 3 ed. São Paulo: Papirus, 2005. MINISTERIO DE EDUCACIÓN (MINED). Carta Metodológica 1/2000. La Habana: MINED, 2000.

MINISTERIO DE EDUCACIÓN SUPERIOR (MES). Reglamento para el Trabajo Docente y Metodológico en la Educación Superior. Resolución No. 269/91. La Habana: MES, 1991.

MINISTERIO DE EDUCACIÓN SUPERIOR. El Problema de la Permanencia en la Educación Superior. La Habana: Dirección de Formación de Profesionales, 2005b.

MINISTERIO DE EDUCACIÓN SUPERIOR. La universidad que queremos. La Habana: Dirección de Formación de Profesionales - Dirección de Universalización, 2004.

MINISTERIO DE EDUCACIÓN SUPERIOR. Positivos y negativos. UAE Formación del Profesional (Curso 2005-2006). La Habana: Dirección de Formación de Profesionales, 2006b.

MINISTERIO DE EDUCACIÓN SUPERIOR. Prioridades de la Dirección de Formación de Profesionales para el Curso 05-06. La Habana: Dirección de Formación de Profesionales, 2005a.

MINISTERIO DE EDUCACIÓN SUPERIOR. Prioridades de la Dirección de Formación de Profesionales. Curso 06-07. La Habana: Dirección de Formación de Profesionales, 2006c.

MINISTERIO DE EDUCACIÓN SUPERIOR. Reglamento para la aplicación de las 
categorías docentes en la educación superior. (Resolución 128). La Habana: MES, $2006 a$. OIE-UNESCO. Calidad de la educación y el papel fundamental de los docentes, 2002. Disponible: <http://www.ibe.unesco.org/international/ICE47/Spanish/Organisation/ Workshops/Background\%20at-4\%20SPA.pdf Acceso 4 de abril de 2006d>.

ORTIZ, A. L. El arte de enseñar: ¿Cómo preparar y desarrollar clases de calidad? Centro de Estudios Pedagógicos y Didácticos (CEPEDID). Barranquilla, 2005. Disponible en: <http://www.monografias.com/trabajos26/clases-de-calidad/clases-de-calidad.shtml $>$. Acceso 28 de abril de 2006.

ORTIZ, E. Un modelo de personalidad para la formación de valores en la educación superior. Universidad de La Habana, 2000. Disponible en: <http://www.psicologiacientifica.com/publicaciones/biblioteca/articulos/arortiz01.htm>. Acceso 28 de abril de 2006

PIMIENTA, Selma G., ANASTASIOU, L. da G. Docência no ensino Superior. 2 ed. São Paulo: Cortez, 2005.

RICO, P.; SILVESTRE, M. Proceso de enseñanza-aprendizaje. La Habana: ICCP, 1997.

SILVESTRE, M. Aprendizaje, educación y desarrollo. La Habana: Pueblo y Educación 1999. SILVESTRE, M.; ZILBERSTEIN, J. Hacia una didáctica desarrolladora. La Habana: Pueblo y Educación, 2002.

ZILBERSTEIN, José T. Categorías en una didáctica desarrolladora. Posición desde el enfoque histórico-cultural. In: Preparación Pedagógica Integral para Profesores Integrales. La Habana: Editorial Félix Varela, 2006, p. 33-43.

\section{NOTAS}

${ }^{1}$ El proyecto "Formación continua de profesores universitarios: desempeño docente, trabajo metodológico y categorización docente" se lleva a cabo entre investigadores del Centro Universitario de Sancti Spiritus (CUSS), Cuba, y el Centro Universitario de Triángulo (Unitri), MG, Brasil, en el marco del convenio de cooperación internacional existente entre ambas IES.

2 Aunque las premisas se sitúan en la Campaña Nacional de Alfabetización de 1961 y en la Reforma Universitaria de 1962, es a partir del año 2000 que se da inicio en Cuba a la construcción de un nuevo modelo de IES que se ha dado en llamar la nueva universidad cubana. El modelo se basa en el libre acceso, para lo cual se han creado Sedes Universitarias Muncipales (SUM) en los 169 municipios del país. Lo que está en perspectiva es la formación de una cultura general integral a escala social, el incremento paulatino de los niveles de equidad y de justicia social, la diversificación de las fuentes de ingreso y la flexibilidad de los modelos pedagógicos, entre otros aspectos organizativos y curriculares. Para una información más exhaustiva, vid. Horruitiner, Pedro. La Universidad Cubana: el modelo de formación. La Habana: Félix Varela, 2006. Passim. También: CD-ROM Memorias de Universidad 2006. La Habana: MES, 2006. Passim.

${ }^{3}$ El modelo cubano de formación descansa en dos ideas rectoras: la unidad entre la instrucción y la educación, y la vinculación del estudio con el trabajo. "La primera de ellas está relacionada con el hecho de que la universidad cubana centra su atención principal... en la formación de los valores que deben caracterizar a ese profesional para que 
pueda servir del mejor modo posible a su país; atendiendo, desde el contenido de cada materia de estudio, cómo se van formando esos rasgos de su personalidad. De hecho, tanto en su concepción teórica, como en su real materialización en el proceso de formación, la unidad de los aspectos educativos con los de carácter instructivo, constituye la idea rectora principal de la educación superior cubana (...) Un segundo aspecto... es la integración que se logra actualmente entre la universidad y la sociedad, que se expresa en la posibilidad de que los estudiantes universitarios cubanos, en todas sus carreras, dediquen una parte importante de su tiempo de estudio a desarrollar habilidades y competencias profesionales en diferentes entidades laborales, productivas y de servicios, a todo lo largo y ancho del país. Ese nexo, que gradualmente se desarrolla y consolida, caracteriza la otra idea rectora principal de la educación superior en Cuba: el vínculo del estudio con el trabajo”. Cfr. Horruitiner, 2006, p.11.

${ }^{4}$ G. F. Shea (1992) nos recuerda que "la historia de los tutores comienza en tiempos de Ulises. Cuando Ulises se va a la guerra de Troya encomienda el cuidado de su hijo Telémaco a un tutor, llamado Mentor (por eso los ingleses han adoptado la denominación de "mentores" para los tutores). Desde esos lejanos antecedentes, la idea de los tutores y mentores ha estado siempre ligada a la de personas de confianza que pueden actuar de consejeros, amigos, maestros o de personas prudentes dispuestas a prestar ayuda”. Apud. Zabalza, M.A., 2006, p. 126.

${ }^{5}$ Al respecto, Vid., por ejemplo: MES. Prioridades de la Dirección de Formación de Profesionales para el Curso 05-06. La Habana: Dirección de Formación de Profesionales, 2005a y MES. Prioridades de la Dirección de Formación de Profesionales. Curso 06-07. La Habana: Dirección de Formación de Profesionales, 2006c.

${ }^{6}$ En el sistema universitario cubano se denominan Profesores principales a los jefes o coordinadores de disciplinas. Las disciplinas son áreas de conocimientos y están integradas por varias asignaturas afines.

${ }^{7}$ En todos los casos los evaluadores trabajaron con la misma Guía de Observación de las Actividades Docentes.

Recebido: 26/06/2007

Aprovado: 05/02/2009

Contato:

Universidade Federal de Uberlândia

Faculdade de Educação/FACED

Av. João Naves de Ávila, 2121

Campus Santa Mônica

Sala $1 G 122$

Uberlândia-MG

CEP 38.408-100 